\title{
Transketolase protein TKTL1 overexpression: A potential biomarker and therapeutic target in breast cancer
}

\author{
MARTHA FÖLDI ${ }^{1}$, ELMAR STICKELER ${ }^{1}$, LIDIJA BAU ${ }^{1}$, OLIVER KRETZ $^{2}$, DIRK WATERMANN ${ }^{1}$, \\ GERALD GITSCH $^{1}$, GIAN KAYSER ${ }^{3}$, AXEL ZUR HAUSEN ${ }^{3}$ and JOHANNES F. COY ${ }^{4}$ \\ ${ }^{1}$ Department of Obstetrics and Gynaecology, University Hospital Freiburg, Hugstetter Str. 55, D-79106 \\ Freiburg; ${ }^{2}$ Anatomy and Cell Biology, University Hospital Freiburg, Albertstr. 17, D-79104 Freiburg; \\ ${ }^{3}$ Institute of Pathology, University Hospital Freiburg, Breisacherstr. 115a, D-79106 Freiburg; \\ ${ }^{4}$ R-Biopharm AG, Landwehrstrasse 54, D-64293 Darmstadt, Germany
}

Received September 4, 2006; Accepted November 20, 2006

\begin{abstract}
Malignant tumors degrade glucose to lactate even in the presence of oxygen via the pentose phosphate pathway (ppp). The non-oxidative part of the ppp is controlled by thiamine-dependant transketolase enzyme reactions. Overexpression of the transketolase-like-1-gene (TKTL1) in urothelial and colorectal cancer is associated with poor patient outcome. We analyzed the expression of the TKTL1 protein in a retrospective institution-based patient cohort with invasive breast cancer by immunohistochemical analysis of 124 paraffin-embedded breast cancer tissues. Our study revealed TKTL1 expression in $86 \%$ of breast cancer specimens with $45 \%$ showing high expression levels. In contrast, only $29 \%$ of corresponding non-neoplastic breast tissues were TKTL1 immunopositive, including 9\% with high expression levels. High expression levels of TKTL1 correlated significantly to Her $2 /$ neu overexpression $(\mathrm{p}=0.015)$. However, TKTL1 expression failed to reach statistical significance for other common prognostic parameters. In contrast to recent data for e.g. colorectal cancer TKTL1 overexpression did not correlate to patient outcome and survival. However, in the context of novel insights into TKTL1-related tumor metabolism and the high proportion of TKTL1 overexpressing breast cancers, this enzyme represents a potential candidate for targeted inhibition of tumor growth in this tumor entity.
\end{abstract}

\section{Introduction}

Malignant cells show complex genetic alterations, which determine their abnormal cellular proliferation, differentiation and survival. Changes in gene expression profiles in malignant

Correspondence to: Dr Axel zur Hausen, Institute of Pathology, University Hospital Freiburg, Breisacherstr. 115a, D-79106 Freiburg, Germany

E-mail: axel.zurhausen@uniklinik-freiburg.de

Key words: Warburg effect, tumor metabolism, breast cancer, transketolase-like-1 cells also modify biochemical pathways, which result in abnormal tumor metabolism.

In most non-neoplastic tissues, glucose is degraded via the Embden-Meyerhof pathway, in case of sufficient oxygen supply and physiological mitochondria activity. If oxygen is absent, healthy cells switch to a fall back reaction in which glucose is fermented to lactate. If oxygen is available, the fermentation is blocked by oxygen, and the intact mitochondria are used for oxidative phosphorylation. This regulation ensures the efficient glucose usage by mitochondrial oxidative phosphorylation in the presence of sufficient oxygen supply.

In contrast, especially in carcinoma tissues glucose is fermented to lactate even if oxygen is present (aerobic glycolysis; 'Warburg effect') (1). Although this particular metabolism in cancer was identified 80 years ago, the relevance of aerobic glycolysis for cancer cell biology is still controversial $(2,3)$. A mutated transketolase enzyme (transketolase-like-1/TKTL1) has been suggested to be a basis of a mammalian glucose fermentation pathway (4).

Transketolase enzyme reactions enable an oxygenindependent glucose degradation and play a crucial role in nucleic acid ribose synthesis utilizing glucose carbons in tumor cells (5). As a consequence, the treatment of tumor cells with specific transketolase inhibitors led to a reduction in tumor cell proliferation (6), and activation of transketolases by application of thiamine stimulated tumor growth (7).

Three human transketolase genes have been recognized: TKT, TKTL1 and TKTL2. TKT is known to encode an active transketolase enzyme (8), and TKTL2 is also likely to encode an active transketolase enzyme. TKTL1 has been assumed to be a pseudogene; however, it has been previously shown that TKTL1 could encode a transketolase-like protein (9). The relative contributions of TKT, TKTL1 and TKTL2 to tumorspecific transketolase metabolism have been investigated in a recent study which found a specific TKTL1 overexpression in urothelial and colon carcimomas at the mRNA and protein level, whereas TKT- and TKTL2-expression are not upregulated. Overexpression of TKTL1 protein predicted poor patient survival (10).

Evidence that breast carcinomas also exhibit the Warburg effect can be derived from a series of previous studies $(11,12)$. 
However, the expression of TKTL1 protein in breast cancer has not yet been investigated.

In the present study, we examined 124 breast cancer samples for specific TKTL1 protein expression by immunohistochemistry. A marked subset of breast cancers showed a TKTL1 overexpression which correlated significantly with Her2/neu overexpression. However, in contrast to colon and urothelial cancer, TKTL1 overexpression was not associated with a poor patient outcome in the examined study population. Due to the high rate of TKTL1 overexpression, we assume that TKTL1 can be viewed as a novel metabolic biomarker in breast cancer, and a possible future target for mechanismdriven drug development.

\section{Materials and methods}

Patient sample and histopathological criteria. A total of 124 breast cancer patients without metastasis or second malignancies treated for invasive breast cancer at the Department of Obstetrics and Gynaecology, University Hospital Freiburg, Germany, between 1990 and 1999 were included into this retrospectively designed study. Breast conserving surgery or mastectomy was performed without prior radio-, chemo- or endocrine therapy.

Adjuvant systemic therapy was given according to the international recommendations of the time. After breast conserving surgery radiation was performed with 52 Gy and a $10 \mathrm{~Gy}$ boost to the tumor area. After mastectomy radiation was given only to advanced tumors. In hormone receptorpositive patients tamoxifen was recommended for a period of 2-5 years. Systemic chemotherapy was mostly given to lymph node positive patients and was mainly CMF (cyclophosphamide, methothrexate, 5-fluorouracil)-based, only in some patients anthracyclines were used for adjuvant treatment.

Immunohistochemistry. For immunohistochemistry (IHC) 3- to $5-\mu \mathrm{m}$ paraffin sections of tissue microarrays of the 124 breast cancers and corresponding non-neoplastic tissues were independently analyzed by two experienced pathologists (G.K., A.z.H.). Antigen demasking was performed by heating dewaxed sections in $10 \mathrm{mM}$ sodium citrate $(\mathrm{pH} \mathrm{6.0)}$ in a microwave oven for $1 \mathrm{~min}$ at $450 \mathrm{~W}$ followed by $5 \mathrm{~min}$ at $100 \mathrm{~W}$. Inhibition of endogenous peroxidase was performed by a 5 min incubation with $3 \% \mathrm{H}_{2} \mathrm{O}_{2}$. Endogenous avidin-biotin was blocked by the use of a commercial biotin blocking system (Dako) for $10 \mathrm{~min}$. After two washes in Tris-saline buffer (TBS), slides were incubated with $1 \%$ goat serum for 30 min to block unspecific staining. Sections were subsequently exposed to mouse anti-TKTL1 (clone JFC12T10; mouse IgG2b) antibody $\left(15 \mu \mathrm{g} \mathrm{ml}^{-1}\right)$ for $1 \mathrm{~h}$. The monoclonal anti-TKTL1 antibody JFC12T10 has been described $(4,10)$. Slides were washed in TBS and incubated with biotinylated anti-mouse immunoglobulins for $30 \mathrm{~min}$ at room temperature and treated with streptavidin-peroxidase (Dako). Staining was revealed using 3-amino-9-ethylcarbazole (AEC) substrate and counter-stained with haematoxylin.

The obtained tissue underwent routine staining for the standard histopathological and prognostic parameters, such as tumor type, tumor size (T), nodal status (N), estrogen/ progesterone receptor expression (ER/PR), grading $(\mathrm{G})$ and
Table I. Histopathological characteristics of included tumors and level of TKTL1 expression.

\begin{tabular}{|c|c|c|c|c|c|}
\hline & & & TKTL1 & xpression & \\
\hline & & $\mathrm{N}$ & Low $(\%)$ & High $(\%)$ & p-value \\
\hline T stage & 1 & 53 & $22(41.5)$ & $31(58.5)$ & 0.052 \\
\hline & 2 & 51 & $34(66.6)$ & $17(33.3)$ & \\
\hline & $3+4$ & 20 & $12(60.0)$ & $8(40.0)$ & \\
\hline Nodal status & 0 & 68 & $33(48.5)$ & $35(51.5)$ & 0.12 \\
\hline & 1 & 56 & $35(62.5)$ & $21(37.5)$ & \\
\hline Grading & $1+2$ & 63 & $32(50.8)$ & $31(49.2)$ & 0.38 \\
\hline & 3 & 61 & $36(59.0)$ & $25(41)$ & \\
\hline ER & Pos & 83 & $45(54.2)$ & $38(45.8)$ & 0.843 \\
\hline & $\mathrm{Neg}$ & 41 & $23(56.1)$ & 18 (43.9) & \\
\hline PR & Pos & 90 & $52(57.8)$ & $38(42.2)$ & 0.285 \\
\hline & $\mathrm{Neg}$ & 34 & $16(47.1)$ & $18(52.9)$ & \\
\hline Her2/neu & $0+1$ & 111 & $65(58.6)$ & $46(41.4)$ & 0.015 \\
\hline & $2+3$ & 13 & $3(23.1)$ & $10(76.9)$ & \\
\hline
\end{tabular}

Her2/neu score (Her2; Dako HercepTest ${ }^{\mathrm{TM}}$ ). To facilitate statistical analysis of TKTL1 expression, these criteria were categorized into expression groups as shown in Table I. In brief, TKTL1 expression was scored on a scale ranging from 0 to 3 : score $0,0-20 \%$, score $1,21-50 \%$, score $2,51-80 \%$, and score $3>80 \%$ of the tumor cells stained for TKTL1.

Hormone receptors and Her2/neu expression. Immunohistochemical staining was quantitatively evaluated as follows: For the estrogen- and progesterone-receptor proteins the percentage of tumor cells with nuclear immunostaining was calculated. Additionally, we applied a staining score according to its intensity: 0, no staining; 1, weak staining intensity; 2 , moderate staining intensity; 3 , strong staining intensity. For Her2/neu, we followed the recommended HercepTest guidelines: score 0 , no membrane staining in tumor cells; 1 , incomplete membrane staining in $>10 \%$ of tumor cells; 2 , weak to moderate complete membrane staining in $>10 \%$ of tumor cells; 3 , complete membrane staining in $>10 \%$ of tumor cells with strong staining intensity.

Statistical analysis. To facilitate statistical analysis, criteria were categorized into expression groups as shown in Table I. TKTL1 scores of 0 and 1 were summarized as low and scores of 2 and 3 were categorized as high expression. To compare both groups the Chi-square test and Fisher's exact test were used. The significance level was set at $5 \%$, and each p-value was two tailed.

Overall survival was defined as time from surgery to death, or the date last known to be alive. Overall survival rates were estimated by the Kaplan-Meier product limit method. The SPSS-software package version 13.0 was used (SPSS for windows, SPSS, Chicago, IL, USA). 


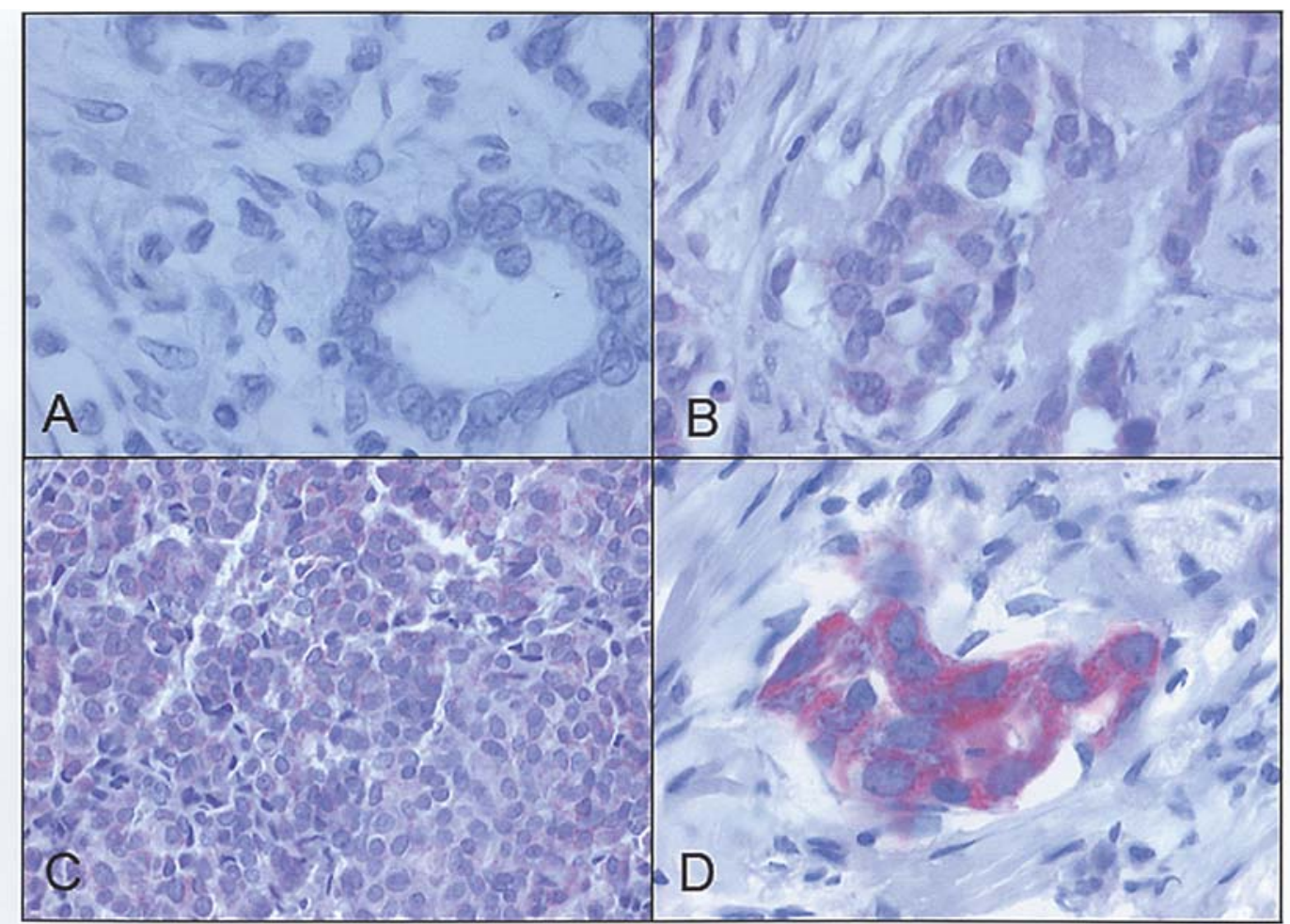

Figure 1. (A) No staining of TKTL1 in epithelial cells of mammary tissue (score 0). (B) Weak cytoplasmic expression (faint red) of TKTL1 in invasive breast carcinoma (score 1). (C) Moderate, specific cytoplasmic expression in an invasive breast carcinoma (score 2). (D) Strong, specific cytoplasmic (red) expression of TKTL1 in an invasive breast carcinoma (score 3).

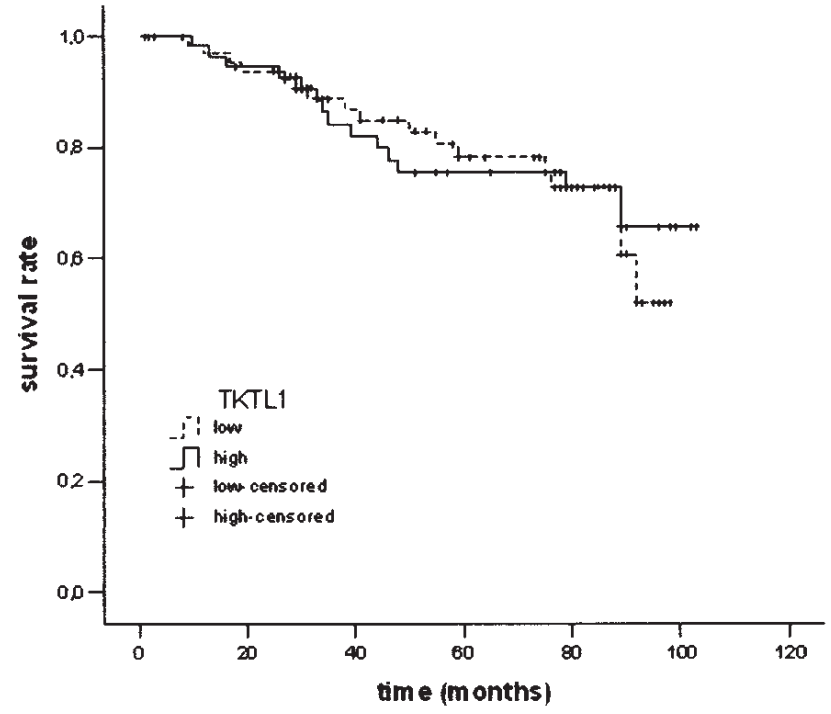

Figure 2. Estimated survival rates by TKTL1 overexpression. Kaplan-Maier curves on overall survival for TKTL1 low (score 0 and 1) and high expression (score 2 and 3 ).

\section{Results}

TKTL1 expression in non-neoplastic tissue and breast cancer. Tumor tissue of 124 patients and corresponding adjacent non-neoplastic breast tissue $(n=41)$ were tested for TKTL1 expression by immunohistochemistry. Analysis of nonneoplastic breast tissue revealed no to weak expression of TKTL1 in 37 samples (90.2\%) with 29 (70.7\%) samples showing score 0 and 8 (19.5\%) samples, score 1, respectively. Only 4 specimens of corresponding normal breast tissues showed high expression levels of TKTL1 (9.8\%).

In contrast, a total of 107 of 124 breast cancers (86\%) expressed TKTL1 specifically in tumor cells. Seventeen specimens $(13.7 \%)$ were TKTL1 negative, whereas $51(41.1 \%)$ showed score 1 . Fifty-six $(45.1 \%)$ breast cancers exhibited a high expression level of TKTL1 with 37 (29.8\%) tumors expressing score 2 and 19 (15.3\%) tumors, score 3 (Fig. 1).

TKTL1 expression and clinico-pathological parameters. We were able to detect a significant positive correlation between Her2/neu status and TKTL1 expression levels: breast cancers with a Her $2 /$ neu score of 2 and 3 displayed a significantly increased TKTL1 expression whereas Her2/neu scores of 0 and 1 correlated with low TKTL1 staining intensity $(\mathrm{p}=0.015$; Table I). However, no significant correlation could be found for TKTL1 expression levels and T stage $(\mathrm{p}=0.052)$, nodal status $(p=0.12)$, grading $(p=0.358)$ or hormone receptor expression (ER/PR; $\mathrm{p}=0.843$ and 0.285 , respectively; Table I).

TKTL1 expression and patient outcome and survival. In order to examine a possible impact of the level of TKTL1 overexpression on patients' overall survival, a Kaplan Meier analysis was performed. We found no correlation of TKTL1 expression to treatment modalities. There was no significant correlation to patients age $(\mathrm{p}=0.346)$, surgical procedure $(\mathrm{p}=0.199)$, adjuvant radiation therapy $(\mathrm{p}=0.849)$, adjuvant chemotherapy $(\mathrm{p}=0.773)$ or endocrine treatment $(\mathrm{p}=0.206)$. Moreover, our analysis did not reveal a correlation of TKTL1 overexpression to overall survival (Fig. 2). 


\section{Discussion}

This study analyzing the expression of TKTL1 in breast cancer revealed an induction of TKTL1 expression in breast cancer from $29 \%$ in corresponding non-neoplastic versus $86 \%$ in neoplastic tissue. The highest score level was determined in $9 \%$ of non-neoplastic and $45 \%$ of breast cancer tissue, respectively.

Our data on TKTL1 expression in non-neoplastic breast tissue are supported by a study which determined TKTL1 expression in various non-neoplastic tissues, especially in those exhibiting increased aerobic glycolysis (4). Since the non-neoplastic tissue specimens are derived from adjacent areas of the invasive breast cancer the observed high expression levels of TKTL1 in a small subset of these nonneoplastic specimens might reflect ongoing alterations in gene expression patterns which accompany the initial steps of breast cancer tumorigenesis. These effects have been described for other genes such as p53, FAS or GLUT1 $(13,14)$.

In the investigated patient cohort we detected a significant positive correlation of TKTL1 and Her2/neu overexpression. In breast cancer, the Her2/neu gene is amplified in $25-30 \%$ of the cancers and is in these cases a negative predictor for patient prognosis $(15,16)$. In our study cohort TKTL1 overexpression failed to show a significant correlation with clinicopathological parameters, other than Her2/neu, such as T stage, nodal status or grading. These results are in contrast to data on colorectal and urothelial cancer where TKTL1 overexpression is associated with aggressiveness of tumors and a poor patient outcome (10). In the present breast cancer patient cohort TKTL1 failed to be a valuable predictive marker. However, it is of importance to note that also Her $2 /$ neu overexpression in our patient cohort also failed to be a negative prognostic predictor (data not shown). However, in the context of metabolic changes observed in malignant tumors and metastases, TKTL1 protein overexpression might be of potential biological, diagnostic and therapeutic significance in breast cancer. Tumor cells with intact mitochondrial oxidative phosphorylation have been experimentally converted to respiration-defect clones by damaging the mtDNA. The altered tumor cells performed aerobic glucose fermentation and were less sensitive to chemotherapeutic agents commonly used in the clinical treatment of cancer, but significantly responded to inhibition of glucose fermentation (17). Activation of the oncogenic kinase Akt has been shown to stimulate glucose uptake and metabolism in cancer cells, and renders these cells susceptible to death in response to glucose withdrawal (18). Such tumor cells have been shown to be dependent on glucose because the ability to induce fatty acid oxidation in response to glucose deprivation is impaired by activated Akt (19). This might explain why ketogenic diet has been successfully applied to children with aggressive brain tumors $(20,21)$.

Metabolic changes in tumor cells might in future be exploited for the development of novel drugs for the inhibition of glucose fermentation. Similar to the relevance of Her2/neu as a 'drugable' target for an individualized anti-cancer therapy (22-25), TKTL1 might also be a future target for small molecule drug design and individualized treatment strategies in breast cancer: metabolic control analysis and inhibition of transketolase enzyme reactions have shown in vitro as well as in vivo that tumor proliferation can be inhibited by antitransketolase approaches (5-7,26-29).

In summary, TKTL1 might represent a novel biomarker and a 'drugable' target in breast cancer. Determination of metabolic changes in tumors via TKTL1 might lead to aprogress in individualized diagnostic and anti-cancer treatment strategies.

\section{References}

1. Warburg O, Posener K, Negelein E: Über den Stoffwechsel der Carcinomzelle. Biochem Z 152: 309-344, 1924.

2. Garber K: Energy boost: the Warburg effect returns in a new theory of cancer. J Natl Cancer Inst 96: 1805-1806, 2004.

3. Gatenby RA and Gillies RJ: Why do cancers have high aerobic glycolysis? Nat Rev Cancer 4: 891-899, 2004.

4. Coy JF, Dressler D, Wilde J and Schubert P: Mutations in the transketolase-like gene TKTL1: clinical implications for neurodegenerative diseases, diabetes and cancer. Clin Lab 51: 257-273, 2005.

5. Boros LG, Puigjaner J, Cascante M, et al: Oxythiamine and dehydroepiandrosterone inhibit the nonoxidative synthesis of ribose and tumor cell proliferation. Cancer Res 57: 4242-4248, 1997

6. Rais B, Comin B, Puigjaner J, et al: Oxythiamine and dehydroepiandrosterone induce a G1 phase cycle arrest in Ehrlich's tumor cells through inhibition of the pentose cycle. FEBS Lett 456: 113-118, 1999.

7. Comin-Anduix B, Boren J, Martinez S, et al: The effect of thiamine supplementation on tumour proliferation. A metabolic control analysis study. Eur J Biochem 268: 4177-4182, 2001.

8. Schenk G, Duggleby RG and Nixon PF: Properties and functions of the thiamin diphosphate dependent enzyme transketolase. Int J Biochem Cell Biol 30: 1297-1318, 1998.

9. Coy JF, Dubel S, Kioschis P, et al: Molecular cloning of tissuespecific transcripts of a transketolase-related gene: implications for the evolution of new vertebrate genes. Genomics 32: 309-316, 1996.

10. Langbein S, Zerilli M, Zur Hausen A, et al: Expression of transketolase TKTL1 predicts colon and urothelial cancer patient survival: Warburg effect reinterpreted. Br J Cancer 94: 578-585, 2006.

11. Isidoro A, Casado E, Redondo A, et al: Breast carcinomas fulfill the Warburg hypothesis and provide metabolic markers of cancer prognosis. Carcinogenesis 26: 2095-2104, 2005.

12. Robey IF, Lien AD, Welsh SJ, Baggett BK and Gillies RJ: Hypoxia-inducible factor-1alpha and the glycolytic phenotype in tumors. Neoplasia 7: 324-330, 2005.

13. Mottolese M, Nadasi EA, Botti C, et al: Phenotypic changes of p53, HER2, and FAS system in multiple normal tissues surrounding breast cancer. J Cell Physiol 204: 106-112, 2005.

14. Alo PL, Visca P, Botti C, et al: Immunohistochemical expression of human erythrocyte glucose transporter and fatty acid synthase in infiltrating breast carcinomas and adjacent typical/ atypical hyperplastic or normal breast tissue. Am J Clin Pathol 116: 129-134, 2001.

15. Slamon DJ, Clark GM, Wong SG, Levin WJ, Ullrich A and McGuire WL: Human breast cancer: correlation of relapse and survival with amplification of the HER-2/neu oncogene. Science 235: 177-182, 1987

16. Revillion F, Bonneterre J and Peyrat JP: ERBB2 oncogene in human breast cancer and its clinical significance. Eur J Cancer 34: 791-808, 1998.

17. Xu RH, Pelicano H, Zhou Y, et al: Inhibition of glycolysis in cancer cells: a novel strategy to overcome drug resistance associated with mitochondrial respiratory defect and hypoxia. Cancer Res 65: 613-621, 2005.

18. Elstrom RL, Bauer DE, Buzzai M, et al: Akt stimulates aerobic glycolysis in cancer cells. Cancer Res 64: 3892-3899, 2004.

19. Buzzai M, Bauer DE, Jones RG, et al: The glucose dependence of Akt-transformed cells can be reversed by pharmacologic activation of fatty acid beta-oxidation. Oncogene 24: 4165-4173, 2005.

20. Nebeling LC and Lerner E: Implementing a ketogenic diet based on medium-chain triglyceride oil in pediatric patients with cancer. J Am Diet Assoc 95: 693-697, 1995. 
21. Seyfried TN and Mukherjee P: Targeting energy metabolism in brain cancer: review and hypothesis. Nutr Metab 2: 30, 2005.

22. Cobleigh MA, Vogel CL, Tripathy D, et al: Multinational study of the efficacy and safety of humanized anti-HER 2 monoclonal antibody in women who have HER2-overexpressing metastatic breast cancer that has progressed after chemotherapy for metastatic disease. J Clin Oncol 17: 2639-2648, 1999.

23. Vogel CL, Cobleigh MA, Tripathy D, et al: Efficacy and safety of trastuzumab as a single agent in first-line treatment of HER2overexpressing metastatic breast cancer. J Clin Oncol 20: 719-726, 2002.

24. Baselga J, Tripathy D, Mendelsohn J, et al: Phase II study of weekly intravenous recombinant humanized anti-p185HER2 monoclonal antibody in patients with HER2/neu-overexpressing metastatic breast cancer. J Clin Oncol 14: 737-744, 1996.

25. Slamon DJ, Leyland-Jones B, Shak S, et al: Use of chemotherapy plus a monoclonal antibody against HER2 for metastatic breast cancer that overexpresses HER2. N Engl J Med 344: 783-792, 2001.
26. Comin-Anduix B, Boros LG, Marin S, et al: Fermented wheat germ extract inhibits glycolysis/pentose cycle enzymes and induces apoptosis through poly(ADP-ribose) polymerase activation in Jurkat T-cell leukemia tumor cells. J Biol Chem 277: 46408-46414, 2002.

27. Du MX, Sim J, Fang L, et al: Identification of novel smallmolecule inhibitors for human transketolase by high-throughput screening with fluorescent intensity (FLINT) assay. J Biomol Screen 9: 427-433, 2004.

28. Boros LG, Lapis K, Szende B, et al: Wheat germ extract decreases glucose uptake and RNA ribose formation but increases fatty acid synthesis in MIA pancreatic adenocarcinoma cells. Pancreas 23: 141-147, 2001.

29. Jakab F, Shoenfeld Y, Balogh A, et al: A medical nutriment has supportive value in the treatment of colorectal cancer. Br J Cancer 89: 465-469, 2003. 